\title{
Extensor pollicis longus tendon rupture as a complication of distal radius fracture: the role of ultrasound examination
}

\author{
Nicola Romano ${ }^{1}$, Aldo Fischetti ${ }^{1}$, Ilaria Mussetto ${ }^{1}$, Marzia Marino², Alessandro Muda ${ }^{3}$
}

${ }^{1}$ Department of Radiology, University of Genoa, ${ }^{2}$ Deparment of Radiology, General Hospital of Loreto, ASUR 2, Ancona, ${ }^{3}$ Department of Radiology, Policlinic San Martino, Genoa, Italy

\section{To the Editor,}

The rupture of the extensor pollicis longus tendon (EPL) is a rare complication of distal radius fracture, firstly described in literature in 1876 by Duplay. The rupture of EPL occurs more frequently in non-displaced or minimally displaced distal radius fractures [1]. The incidence of this complication is variable from $0.3 \%$ to $5 \%$ and more frequently occurs in women, usually between 6.6 weeks after distal radius fracture [1]. There are multiple etiologies that explain the physiopathology of extensor pollicis longus tendon rupture after closed treatment of distal radius fracture. The two major theories that have been proposed are mechanical and vascular. The mechanical theory explains that EPL courses in a vulnerable position around the Lister's tubercle and when fracture occurs, the bone irregularity of the distal radius abrades the tendon, predisposing to rupture [2]. The vascular theory says that the bloody supply of EPL tendon around Lister's tubercle is minor. The increased pressure inside the tendon sheath at Lister's tubercle, due to edema or hematoma secondary to distal radius fracture, further reduces tendon vascularization, resulting in tendon degeneration and delayed rupture [2].

Ultrasound examination usually starts with the identification of the third extensor tendon compartment that is medial or above to the Lister's tubercle. The study in sagittal plane is fundamental to fully observe changes in tendon thickness and tendon stump. The contralateral side

Received 19.12.2017 Accepted 29.12.2017

Med Ultrason

2018, Vol. 20, No 1, 114-115, DOI: 10.11152/mu-1380

Corresponding author: Nicola Romano, MD,

Department of Radiology,

University of Genoa, Italy

Via Leon Battista Alberti 4- 16132- Genoa- Italy

Phone: +393356321624

E-mail: romano.nicola@live.it

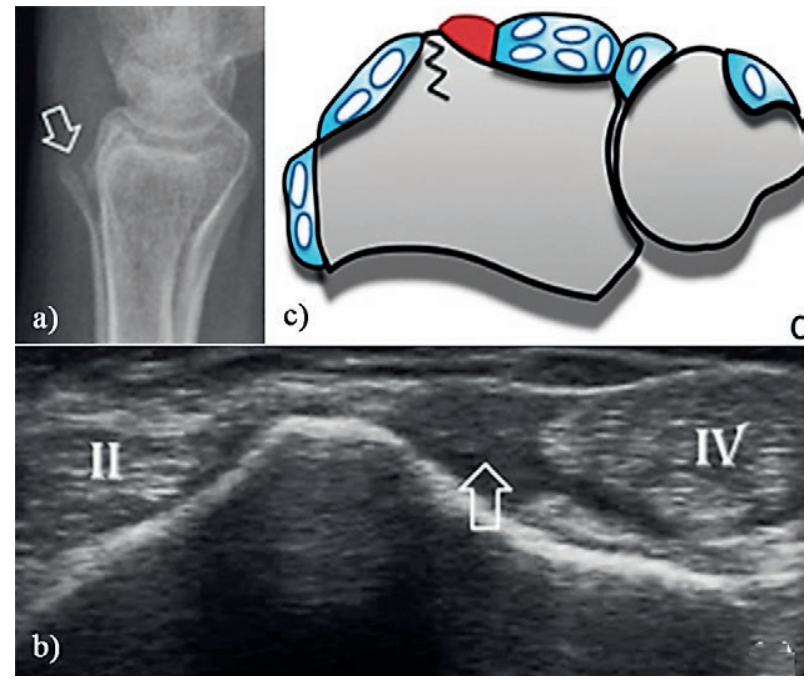

Fig 1. Woman, 55 years-old: a) lateral radiograph of the left wrist showed a non displaced fracture of the distal radius in proximity to the Lister's tubercle (arrow); b) five weeks later ultrasound examination in axial plane, at Lister's tubercle, demonstrated the absence of the EPL tendon (white arrow), between the II and IV extensor tendon compartments; c) schematic drawing of the dorsal aspect of the wrist with rupture and absence of EPL tendon (red compartment) after distal radius fracture at Lister's tubercle.

is used as control for the comparison of tendon thickness and muscle integrity $[3,4]$.

Ultrasound pathologic pattern consists of a gap between tendon stumps occupied by an attenuated hypoechoic string (fig 1). Sonography is very useful in an accurate diagnosis and to determine the position and the degree of the retracted ends of the tendon, and is essential to establish the site of the surgical incision, which should be as small as possible $[3,4]$. Dynamic maneuvers in the sagittal plane on the dorsal aspect of the wrist and thumb, with passive flexion and extension movements, can facilitate the diagnosis. 
Ultrasound examination is not the only technique to study EPL interruption. MRI can also reveal tendon rupture and may allow measurement of the distance between tendon stumps. The two main limitations of MRI are to include the entire EPL muscle and tendon because it may require a double examination, and the magic angle artifact caused by the oblique orientation of the EPL and its flat shape [4].

In conclusion, we want to recall the existence of EPL rupture as rare complication of distal radius fracture and underline the importance of ultrasound examination that is more useful and rapid than other imaging techniques to diagnose EPL tendon tear for preoperative planning and is the best technique to estimate tendon stump separation.

\section{References}

1. Roth KM, Blazar P, Earp B, et al. Incidence of extensor Pollicis longus tendon rupture after nondisplaced distal radius fractures. J Hand Surg Am 2012;37:942-947.

2. Kim CH. Spontaneous Rupture of the Extensor Pollicis Longus Tendon. Arch Plast Surg. 2012;39:680-682.

3. De Maeseneer M, Marcelis S, Osteaux M, et al. Sonography of a rupture of the tendon of the extensor pollicis longus muscle: initial clinical experience and correlation with findings at cadaveric dissection. AJR Am J Roentgenol 2005; 184:175-179.

4. Santiago FR, Plazas PG, Fernández JM. Sonography findings in tears of the extensor pollicis longus tendon and correlation with CT, MRI and surgical findings. Eur J Radiol 2008;66:112-116.

\title{
Ultrasound guided injection for medial and lateral pterygoid muscles: a novel treatment for orofacial pain
}

\author{
YunnJy Chen ${ }^{1}$, Pei-Hsuan Chang ${ }^{1}$, Ke-Vin Chang², Wei-Ting Wu², Levent Özçakar ${ }^{3}$
}

${ }^{1}$ Department of Dentistry, School of Dentistry, National Taiwan University Hospital Taipei, Taiwan, ROC, ${ }^{2}$ Department of Physical Medicine and Rehabilitation and Community and Geriatric Research Center, National Taiwan University Hospital, Bei-Hu Branch and National Taiwan University College of Medicine, Taipei, Taiwan, ${ }^{3}$ Department of Physical and Rehabilitation Medicine, Hacettepe University Medical School, Ankara, Turkey

\section{To the Editor}

Temporomandibular myofascial pain has a prevalence ranging between $17 \%$ and $19 \%$ in the general population $[1,2]$. It substantially stems from the masticatory muscles two of which are medial (MPM) and lateral pterygoid muscles (LPM). The MPM has two origins; the medial aspect of the lateral pterygoid plate and the maxillary tuberosity of the palatine bone. Its insertion is on the medial surface of the mandibular bone. Likewise,

Received 07.12.2017 Accepted 28.12.2017

Med Ultrason

2018, Vol. 20, No 1, 115-116, DOI: 10.11152/mu-1362

Corresponding author: Ke-Vin Chang, MD, PhD

Department of Physical Medicine and

Rehabilitation, National Taiwan University

Hospital, Bei-Hu Branch, No. 87 Neijiang St,

Wanhua District, Taipei City 108, Taiwan.

E-mail: kvchang011@gmail.com; pattap@pchome.com.tw the LPM originates from the greater wing of the sphenoid bone and the lateral surface of the lateral pterygoid plate and inserts to the condylar process of the mandible. While contraction of the former results in mouth closure, contraction of the latter leads to mouth opening. Both muscles play an important role in the development of orofacial pain $[3,4]$; however, blind injections carry a significant risk of neurovascular injury. Therefore, in this report, we propose two ultrasound-guided approaches and also test their feasibility on the water phantom models.

The curvilinear transducer is more preferable than the linear one, considering the depth of both targets. For the MPM injection, the patient lies down with the head extended to expose the chin where the transducer is placed in the coronal plane. From superficial to deep layers lie the suprahyoid and glossus muscles, the tongue and the oral cavity with the palate, appearing as a hyperechoic flattened structure at the bottom. Relo- 


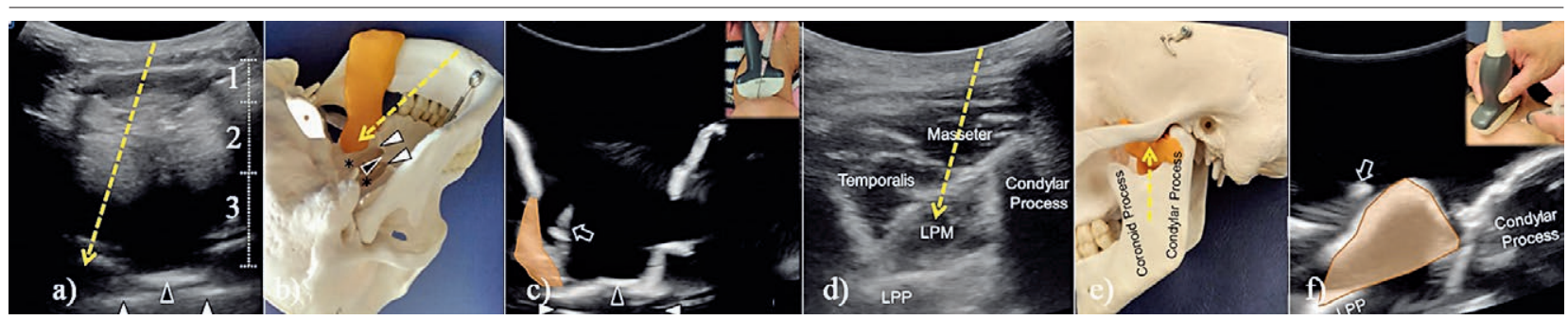

Fig 1. a) Ultrasound imaging for the medial pterygoid muscle with the transducer placed on the chin in the coronal plane (1 - suprahyoid and glossus muscles, 2 - tongue, 3 - oral cavity); b) A skull model for demonstrating the medial pterygoid muscle; c) Ultrasound guided injection to the medial pterygoid muscle on the skull model in the water; d) Ultrasound imaging of the lateral pterygoid muscle with the transducer placed below the zygomatic arch in the horizontal plane; e) A skull model for demonstrating the lateral pterygoid muscle; f) Ultrasound guided injection to the lateral pterygoid muscle on the skull model in the water. Black arrowhead posterior nasal spine; white arrowhead - nasal choana; dashed yellow arrow - imaginary needle trajectory; black arrows - needle tip. All the ultrasound images were acquired by using a curved transducer (Ecube 15; Alpinion, Seoul, Korea).

cating the transducer posteriorly towards the neck allows the visualization of the posterior nasal spine in the midline with both nasal choanas nearby (fig 1a). The sonographic appearance is similar to a mountain range, with the posterior nasal spine as the peak and the nasal choanas as the valleys. The needle can be inserted through the center of the transducer in an out-of-plane trajectory, targeting the muscular wall lateral to the nasal choana, where the MPM is attached (fig 1b,c; Video 1 , on the journal site). The needle should not be inserted deeper than the level of the posterior nasal spine. Otherwise, the needle path may be blocked by the medial pterygoid plate.

For LPM injection, the patient is side-lying and the transducer is placed parallel to the zygomatic arch. The transducer is moved caudally to bridge the mandibular notch between the coronoid and condylar processes. Opening the mouth relocates the coronoid process more caudally to create a gap distal to the zygomatic arch. Through the window, the LPM can be fully depicted as a triangular muscle originating from the condylar process and inserting to the lateral pterygoid plate (fig 1d). The needle is inserted through the midline of the transducer by using the out-of-plane approach targeting the LPM (fig 1e,f; Video 1, on the journal site). Before the injection, the power Doppler mode should be turned on to avoid incidental injury of the maxillary artery coursing between the LPM and the temporalis muscles.
In short, the present report demonstrates that the ultrasound guided techniques can be feasible and precise for the treatment of orofacial pain originating from the pterygoid muscles.

Acknowledgments: the present study is supported by National Taiwan University Hospital, Bei-Hu Branch, Ministry of Science and Technology (MOST 106-2314B-002- 180-MY3) and Taiwan Society of Ultrasound in Medicine.

\section{References}

1. Jivnani HM, Tripathi S, Shanker R, et al. A study to determine the prevalence of temporomandibular disorders in a young adult population and its association with psychological and functional occlusal parameters. J Prosthodont 2017, doi: 10.1111/jopr.12704

2. Marklund S, Wanman A. Incidence and prevalence of myofascial pain in the jaw-face region. A one-year prospective study on dental students. Acta Odontol Scand 2008;66:113121.

3. Chen H, Whittle T, Gal JA, Murray GM, Klineberg IJ. The medial pterygoid muscle: a stabiliser of horizontal jaw movement. J Oral Rehabil 2017;44:779-790.

4. Desmons S, Graux F, Atassi M, Libersa P, Dupas PH. The lateral pterygoid muscle, a heterogeneous unit implicated in temporomandibular disorder: a literature review. Cranio 2007;25:283-291 


\title{
Ultrasound to depict anatomical abnormality: an example of potential alliance of rehabilitation professionals.
}

\author{
Daniele Coraci ${ }^{1}$, Arianna Cruciani ${ }^{1}$, Silvia Giovannini ${ }^{2}$, Andrea Bernetti ${ }^{3}$, Valter Santilli ${ }^{3,4}$, \\ Luca Padua ${ }^{1,5}$
}

${ }^{1}$ Don Carlo Gnocchi Onlus Foundation, Milan, ${ }^{2}$ Rehabilitation Units, Policlinico Universitario Agostino Gemelli Foundation, Rome, ${ }^{3}$ Physical Medicine and Rehabilitation Unit, Azienda Policlinico Umberto I, Rome, ${ }^{4}$ Board of Physical Medicine and Rehabilitation, Department of Orthopaedic Science, "Sapienza" University, Rome, ${ }^{5}$ Department of Geriatrics, Neurosciences and Orthopaedics, Catholic University of the Sacred Heart, Rome, Italy
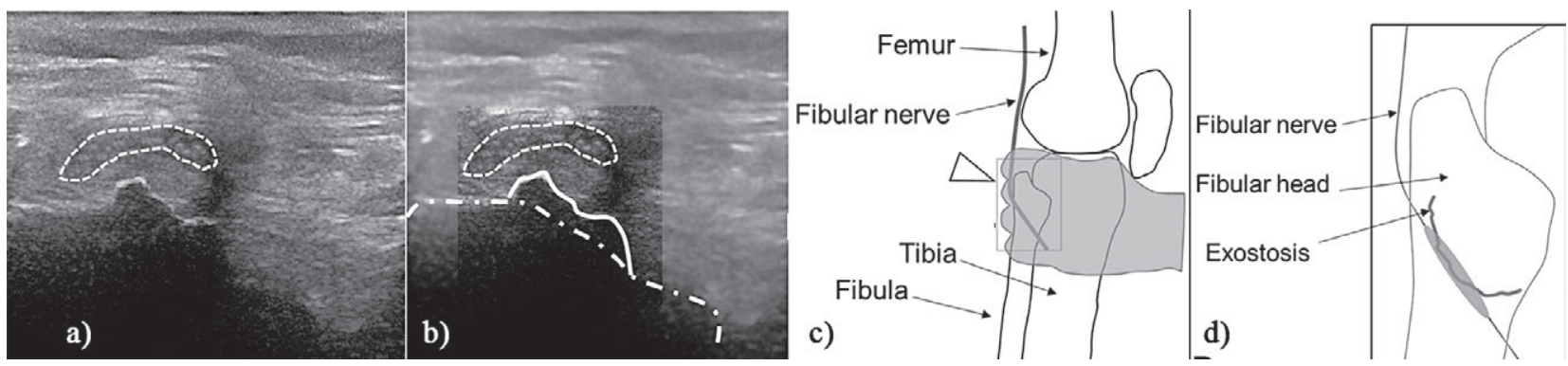

Fig 1. a) Transverse ultrasonographic scan of the fibular nerve (dashed line) at fibular head level, showing increased nerve cross sectional area (15 mm2, normal value up to $12 \mathrm{~mm} 2)$; b) schematic representation of the picture in a), showing the nerve (dashed line) and the exostosis (continuous line) originating from the fibular head (dash-dotted line); c) scheme of the fibular nerve course at knee region. The transparent hand indicates the point where the thrust manipulation was performed; the triangle indicates the site of the ultrasonographic finding; d) zoom-in on a portion of the picture in c): fibular nerve enlargement at the fibular head, where the exostosis is present.

\section{To the Editor,}

Ultrasound (US) application in the rehabilitation field is relatively wide, but US utility should be exploited. The technique may be useful in planning an apparently less invasive therapy, like mobilization [1,2]. Mobilization represents a possible conservative treatment for different musculoskeletal disorders and is commonly considered safe. However, possible side effects are not completely lacking. The knowledge of the patient's anatomy seems a decisive point, because possible anatomical variants could add risk factors for unexpected manipulation-related side effects [3].

Received 22.11.2017 Accepted 30.12.2017

Med Ultrason

2018, Vol. 20, No 1, 117-118, DOI: 10.11152/mu-1342

Corresponding author: Daniele Coraci, MD

Don Carlo Gnocchi ONLUS Foundation

Piazzale Morandi 6, 20121 Milan, Italy

Phone: +39-06-3015-6623

Fax: +39-06-3550-1909

E-mail: danielecoraci@aol.com
We present a 67 year old woman complaining of left foot drop that had occurred one month before our evaluation, after a single session of manual therapy. A thrust maneuver was performed to treat sciatica, applying the force on the left fibular head, although the patient had been complaining of severe pain during manipulation [4]. Our clinical evaluation revealed plegia of the left tibialis anterior muscle (TA). We performed neurophysiological examination, showing a severe axonal damage of the left fibular nerve. US revealed an increased cross sectional area of the nerve at the fibular head $\left(15 \mathrm{~mm}^{2}\right)$ and an anatomical abnormality of the fibular bone: an exostosis, originating from the fibular head, close to the fibular nerve (fig 1). Probably, this alteration, unknown by the therapist and impossible to diagnose without an accurate imaging evaluation, had contributed to the nerve compression during the intense maneuver, with the consequent damage.

Our case confirms a possible side effect of manipulative treatment. Furthermore, it shows how the knowl- 
edge of the anatomical abnormalities, like exostosis, may have been very helpful in avoiding the nerve suffering. US may be considered efficient in depicting the unusual anatomy of a patient and in limiting the potential risky conditions. In our case, the presence of the exostosis and its relationship with the fibular nerve, could have led in considering a different approach [2]. A continuous communication between the physician and therapist should be recommended in similar cases and the manual therapist should ask to the physician all the needed information, so that the treatment can be safely administered. Patient management, especially in the rehabilitation field, involves specific abilities of different healthcare professionals [5]. Merging these skills represents the power of rehabilitation. To obtain this integration, the dialogue among the experts is needed and a decision about the treatment should be shared. In this context, ultrasound, showing patient's anatomy, can be a very useful tool supporting the decision about the most appropriate treatment approach.

\section{References}

1. Berghea F, Vlad V, Palanciuc L, et al. Quantitative Doppler in musculoskeletal ultrasonography - suboptimal performance of both experienced and in-training sonographers in selection of the highest Doppler signal image from cineloops. Med Ultrason 2017;19:282-287.

2. Onat SS, Özişle Z, Orhan A, Akman B, Köklü K, Özçakar L. Ultrasonographic diagnosis of heterotopic ossification and secondary nerve entrapments in a patient with spinal cord injury. Med Ultrason 2017;19:338-339.

3. Grant KE. Massage safety: injuries reported in Medline relating to the practice of therapeutic massage - 1965-2003. J Bodyw Mov Ther 2003; 7:207-212.

4. Santilli V, Beghi E, Finucci S. Chiropratic manipulation in the treatment of acute back pain and sciatica with disc protusion: a randomized double-blind clinical trial of active and simulated spinal manipulations. Spine J 2006;6:11-17.

5. Scott KR, Ahmed A, Scott L, Kothari MJ. Rehabilitation of brachial plexus and peripheral nerve disorders. Handb Clin Neurol 2013;110:499-514. 